\title{
Results From Portugal's 2016 Report Card on Physical Activity for Children and Youth
}

\author{
Jorge Mota, Manuel João Coelho-e-Silva, Armando M. Raimundo, and Luís B. Sardinha
}

\begin{abstract}
Background: This article describes the procedures and development of the first Portuguese Report Card on Physical Activity in Children and Adolescents. Methods: Comprehensive searches for data related to indicators of physical activity (PA) were completed by a committee of physical activity and sports specialists. Grades were assigned to each indicator consistent with the process and methodology outlined by the Active Healthy Kids Canada Report Card model. Results: Nine indicators of PA were graded. The following grades were assigned: Overall Physical Activity Levels, $D$; Organized Sport Participation, $B$; Active Play, $D$; Active Transportation, $C$; Sedentary Behaviors, $D$; Family and Peers, $C$; Schools, $B$; Community and the Built Environment, $D$; and Government, $C$. Conclusions: Portuguese children and adolescents do not reach sufficient physical activity levels and spend larger amounts of time spent in sedentary behaviors compared with recommendations. Effective policies of PA promotion and implementation are needed in different domains of young people's daily lives.
\end{abstract}

Keywords: child health, advocacy, surveillance

In Portugal, monitoring the adherence to recommendations for physical activity (PA) has been conducted mainly by questionnaire $^{1}$ and fewer data are available that use objective measures at the population level. ${ }^{2}$ However, available data from international European studies placed Portugal as one of the highest sedentarylevel countries with a low compliance of the recommended PA levels. ${ }^{3,4}$ In addition, nation-wide studies in children and adolescents are still needed since available results are not consistent, in part due to variation in protocols and data management. Indeed, robust surveillance data for Portugal that would address these needs are lacking. Therefore, it is important to evaluate the state of PA in Portugal and to develop a clear understanding of the problems related to physical inactivity, monitor national trends, and define and plan effective interventions.

The Active Healthy Kids Report Card originated in Canada and is an exceptional tool for presenting the data in a format that is useable by researchers, policy makers, and practitioners. ${ }^{5}$ The Portuguese Report Card on Physical Activity has not been evaluated to date. A systematic evaluation of the factors that affect physical activity behaviors in Portuguese youngsters is essential and can be useful for public health strategies targeting enhanced PA levels.

The current article aimed to describe the procedures and results of the first Portuguese Report Card for Physical Activity in Children and Adolescents. We analyzed relevant data from 2010 to 2016 and searched the references in PubMed as well as the more relevant governmental documents addressing this issue.

Mota is with the Research Centre in Physical Activity, Health and Leisure, Faculty of Sports, University of Porto, Portugal. Silva is with the Faculty of Sport Sciences and Physical Education, University of Coimbra, Portugal. Raimundo is with the Departamento de Desporto e Saúde, Escola de Ciências e Tecnologia; Universidade de Évora, Research Center in Sports Sciences, Health and Human Development (CIDESD), Portugal. Sardinha is with the Exercise and Health Laboratory, Faculty of Human Kinetics, Universidade de Lisboa, Portugal. Mota (jmota@fade.up.pt) is corresponding author.

\section{Methods}

The Research Center in Physical Activity, Health, and Leisure (CIAFEL) located in the Faculty of Sport Sciences at the University of Porto (FADEUP) led and coordinated the Portuguese 2016 Report Card. A 4-member committee was established to prepare the Report Card. The team included researchers in the field of sport and physical activity representing institutions at the national level.

The Portuguese Report Card followed the procedures used in the Active Healthy Kids Canada model..$^{5}$ The sources and availability of evidence were discussed. The group decided that there was sufficient evidence to proceed with the report card. The first phase involved all committee members who collected and discussed available databases, websites, official reports, and contacts at governmental agencies and nongovernmental organizations that are part of education, sports, youth, and health systems. The group had 4 meetings. In the first meeting the research work group met to evaluate the evidence and discuss the grades, which were set after considering the quality of the evidence, the sample size, and the year of the study (2010-2016). Prior to each subsequent meeting, an assigned member showed a grade on the best available evidence. During the meetings the expert group critically discussed the grade. A matrix was then developed and final grades were discussed during the fourth meeting. Grades were assigned to each PA indicator based on estimates of the proportion of children and adolescents that were meeting a specified benchmark or guideline as described elsewhere. ${ }^{5}$

The steering committee developed an idea for the cover of the Portuguese Report Card. Potential ideas were presented at the indicator and grade assignment meetings, and a decision was made during the last meeting. The cover story ("MOVE FOR FUN—BE ACTIVE, BE HEALTHY") is illustrated in Figure 1.

\section{Results}

The 2016 Portuguese Report Card is the first assessment of PA and includes 9 indicators of PA as suggested in the Canadian model ${ }^{5}$ : Overall Physical Activity Levels, Organized Sport Participation, 
Active Play, Active Transportation, Sedentary Behaviors, Family and Peers, School, Community and the Built Environment, and Government. The grades assigned to each indicator are summarized in Table 1.

\section{Discussion}

Portuguese children and adolescents seemed to attain a low level of PA intensities, in addition to larger amounts of time spent in sedentary behaviors, compared with recommendations. ${ }^{2}$ Therefore,

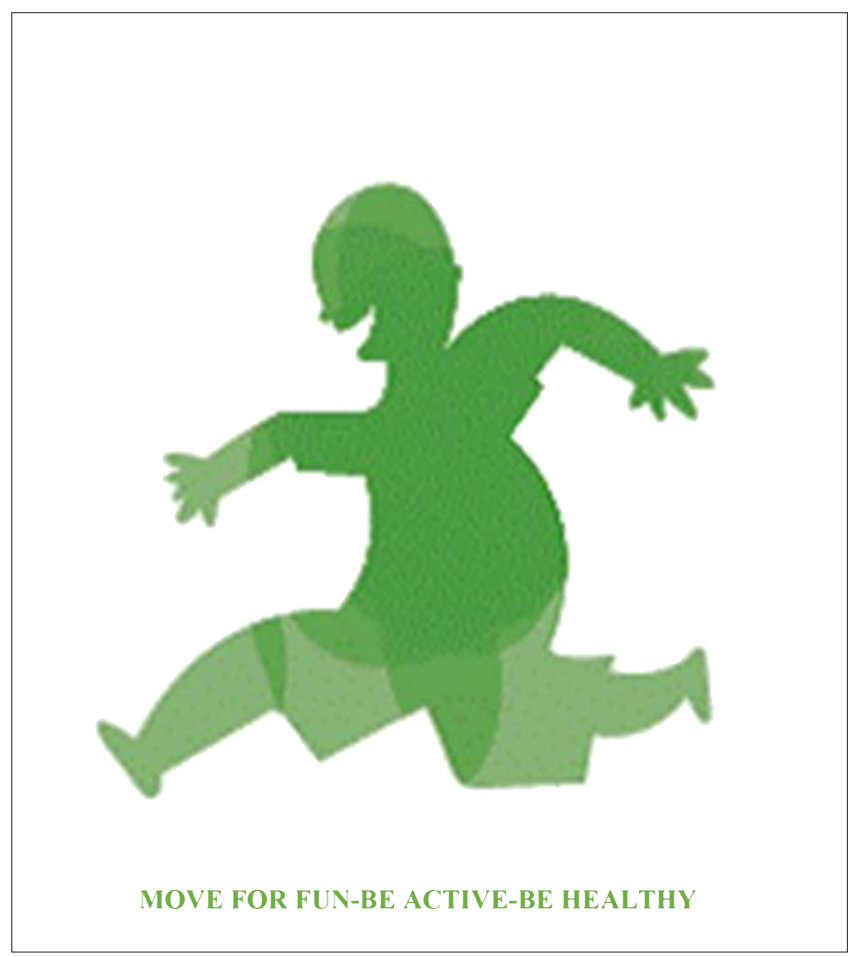

Figure 1 - Front cover of Portugal's 2016 Report Card on Physical Activity for Children and Youth.

Table 1 Grades According to Physical Activity Indicator in the 2016 Portuguese Report Card on Physical Activity for Children and Youth

\begin{tabular}{lc}
\hline Indicator & Grades \\
\hline Overall Physical Activity Levels & $D$ \\
Organized Sport Participation & $B$ \\
Active Play & $D$ \\
Active Transportation & $C$ \\
Sedentary Behaviors & $D$ \\
Family and Peers & $C$ \\
School & $B$ \\
Community and Built Environment & $D$ \\
Government Strategies and Investments & $C$ \\
\hline
\end{tabular}

Note. The grade for each indicator is based on the percentage of children and youth meeting a defined benchmark: $A$ is $81 \%$ to $100 \%, B$ is $61 \%$ to $80 \%, C$ is $41 \%$ to $60 \%, D$ is $21 \%$ to $40 \%, F$ is $0 \%$ to $20 \%$, and $I N C$ is incomplete data. effective policies of PA promotion are needed based on available evidence. Portugal is currently developing national recommendations on health-related PA. The report card provides a unique overall picture of indicators of different forms of PA as well as factors affecting the effectiveness of PA programs at the policy, environmental, and social levels.

\section{Move for Fun-Be Active, Be Healthy}

Drawing upon emerging evidence of the important relationships between physical activity and sedentary behavior and health outcomes, the Portuguese cover story ("Move for Fun-Be Active, Be Healthy") drew attention to the evidence base for the Guidelines with particular reference to research that showed a connection between higher levels of PA associated with playing and having fun leading to higher levels of satisfaction as well as improvements of self-esteem and motivation to be active.

\section{Overall Physical Activity Levels: D}

Overall Physical Activity Level was assigned a grade of $D$. Data are not totally consistent about overall levels of PA. Information obtained from questionnaires, such as the HSBS study, ${ }^{6}$ suggested higher levels of PA compared with information derived from studies using objective assessment of PA, ${ }^{2}$ including governmental reports. ${ }^{7}$ Indeed, data from the Health Behavior in School-aged Children study involving 35 countries in Europe and North America, revealed only $34 \%$ of youth met PA guidelines and placed Portugal in the bottom quartile of countries that satisfied this criteria. ${ }^{8}$ According to this report, only $34 \%$ of Portuguese boys and $17 \%$ of Portuguese girls aged 11 to 15 years were sufficiently active. Similarly, another study involving Portuguese youth found that $36 \%$ of 10 - to 11 -yearolds and $4 \%$ of 16- to 17-year-olds met PA guidelines after using an accelerometer for 4 consecutive days. ${ }^{2}$ Thus, available data showed that health-related PA guidelines were not being reached by a substantial number of youths.

\section{Organized Sport Participation: $B$}

Organized Sport Participation was assigned a grade of $B$. Participation in school sports reached $85 \% .^{6,9}$ At the club level (organized competitive sport) the available data clearly showed an increase of sport participants in clubs. Data obtained from The Portuguese Institute of Sports and Youth (Instituto Português do Desporto e Juventude; IPDJ) registered an increase of 31,000 children engaged in sport federations from 2010-2014. ${ }^{10}$ Statistics relating to sports clubs, athletes, coaches, and participation rates by years and federations were collected, organized, and published by the IPDJ.

\section{Active Play and Leisure: $\boldsymbol{D}$}

Active Play and Leisure was assigned a grade of $D$. Although there were very few available literature related to active play and leisure activities among Portuguese school-aged children, data from the HBSC report showed that $49 \%$ of Portuguese children and adolescents aged 12 to 18 years regularly participated in physical activities outside of school and $14 \%$ did so at least once per week. ${ }^{6}$ Despite the steadily increase of organized sport participation during the last few years the evidence showed that active play during leisure as well as nonorganized sport activities seems to be reducing due to increasing access to technology and other activities. 


\section{Active Transport: $\mathbf{C}$}

A recent study of 1067 urban school children and adolescents showed that $45 \%$ were associated with active transport to and from school. ${ }^{11}$ It was also possible to identify walking as the main pattern of transportation to school while cycling was found to be unusual in Portuguese children. ${ }^{12}$ Additionally, in countryside regions it was found that around $30 \%$ of children $(n=3000)$ aged 7 - to 8-years-old commuted either by foot or by cycling on a regular basis during school days ${ }^{13}$ ). Distance emerged as a significant predictor of active commuting to school as increased distances were associated with higher levels of passive transportation to school. Results also indicate that $2.0 \mathrm{~km}$ seems to be a reasonable maximum distance for walking between home and school. ${ }^{14}$ However, another study showed that of the children that live more than $1 \mathrm{~km}$ away from the school, only $30 \%$ returned home independently and only $11 \%$ returned home actively and independently. ${ }^{15}$ Nonetheless, on average, a walking round trip home-school-home represented $40 \%$ of recommended daily moderate-to-vigorous PA. ${ }^{14}$ Despite the absence of national legislation promoting active travel to schools, a couple of local initiatives (essentially from municipalities) are implementing programs to enhance cycling as a main form of transportation to schools. A grade of $C$ was thus assigned to Active Transport.

\section{Sedentary Behavior: $\boldsymbol{D}$}

A study of children and adolescents aged 10 to 18 years suggested that more than $60 \%$ who were objectively assessed using accelerometers tended to exceed the recommendations for daily sedentary behavior. ${ }^{2,7}$ In addition, several studies reporting time watching TV showed that $20 \%$ and $47 \%$ of Portuguese youth watched TV for $>4$ hour $\cdot$ day $^{-1}$ during weekdays and weekends, respectively. ${ }^{6}$ Popularization of mobile phones and new forms of electronic-based social interactions are contributors to new juvenile cultures. Thus, a grade of $D$ was assigned.

\section{Family and Peers: C}

The importance of family and peer support for the promotion of PA is universally recognized. ${ }^{16}$ However, this indicator is very difficult to examine based on a lack of empirical data adhering to the grading framework. ${ }^{5}$ Indeed, familial determinants and correlates of sport and physical activity were not systematically studied in national representative surveys. Available data were obtained from the Eurobarometer 2014, ${ }^{3}$ where it was pointed out that $9 \%$ of adults in Portugal reported that they engaged in vigorous-intensity PA on at least 4 days, with $23 \%$ of those doing so for $<30$ minutes, $40 \%$ for 31 to 60 minutes, and $23 \%$ for 91 to 120 minutes per day. Moreover, $14 \%$ of adults reported that they engaged in moderate-intensity PA on at least 4 days within the past 7 days, with $34 \%$ of those doing so for $<30$ minutes, $43 \%$ for 31 to 60 minutes, and $15 \%$ for 91 to 120 minutes per day. ${ }^{3}$ Among 2661 Portuguese nuclear families (10,644 subjects), it was suggested that fathers and mothers had a similar influence on their offspring's PA levels irrespective of their sex, and equal sibling correlations point toward shared PA habits. ${ }^{17}$ Further, a similar study, which aimed to investigate predictors and sources of variance of PA levels in nuclear families, suggested a strong dyadic resemblance in PA and showed different effects of gender, age, and BMI on individuals' PA. ${ }^{18}$ Indeed, parental influences on children's mobility has been found. For instance, parental perception of neighborhood safety and parents' self-reported PA might be associated with children's independent mobility. ${ }^{19}$ Moreover, parental education was negatively associated with children's daily
PA patterns and compliance with guidelines. ${ }^{20}$ Considering all the available information, a grade of $C$ was assigned to this category.

\section{School: B}

Physical Education (PE) is mandatory for all Portuguese students until 12th grade. Usually the time allocated to compulsory classes ranges between 90 to 135 minutes per week, although its academic importance decreases over time. Despite the fact that few schools do not have sports facilities and available and adequate equipment, $\mathrm{PE}$ is believed to marginally promote health-related adaptations; but along with sport activities in school it might provide additional support for meeting the recommended PA levels. ${ }^{21}$ The overall analysis is that $\mathrm{PE}$ is provided to a great proportion of children and adolescents. In almost all schools and academic grades, PE classes are provided by specialists in PE. In addition, as mentioned before, schools provide the participation in school sport activities for all students. Thus, a grade of $B$ was assigned for the School indicator.

\section{Community and the Built Environment: $\boldsymbol{D}$}

A grade of $D$ was assigned for the community and the built environment. Indeed, built environmental interventions have been recommended by international authorities for effective promotion of PA behaviors among youth. ${ }^{8}$ Large-scale studies addressing this issue among Portuguese youngsters are still scarce and future research in the topic is needed. The available data showed that perceptions of environmental correlates are based on gender and behavior, ${ }^{11}$ specifically: a) higher levels of overall PA were predicted from better perceptions of sports equipment at home, both in boys and girls; b) boys' PA was significantly associated with aesthetics within the neighborhood; c) active transport to school was associated with safety issues in girls. Nowadays, it is possible to find some good examples of developing facilities and parks viewed as open access environments for PA in parallel to increasing cases of services offering individual and group tutorials of exercise prescription. For instance, parks and availably of bus stops were positively associated with PA among adolescents. Further, parks having recreational facilities and good environmental quality, amenities, and security are related with increased PA among female adolescents. ${ }^{22}$ Additional information about cycling path strategies in municipalities has been developed. ${ }^{23}$

\section{Government: $\mathbf{C}$}

A grade of $C$ was assigned to this indicator. There are several government laws and regulations intended to promote participation in PA and sport. The IPDJ has adopted a National Sports for All Program. ${ }^{24}$ The program defines a set of goals, ultimately aiming to create a strong sporting base to promote and develop sports and education in a Sports for All context. The vision is for all citizens to live healthier lifestyles and enjoy greater quality of life. The plan emphasizes the importance of using sports as a means to bring together the population from all walks of life and from various settings (schools, clubs, companies, and even prisons). The Ministry of Health has created a program on the integration of sports policies into the Portuguese National Health Plan (extended to the year 2020) with a special focus on policy strategies for healthy lifestyles. ${ }^{25}$ In addition, the School Sport Council established sport activities as mandatory for all schools. Broadly speaking, levels of PA and practice of sport have not been impaired by a lack of regulations. Although the policies have not created all of the necessary conditions, they did establish 
an official position that recognizes PA as an important factor in public health and youth development.

\section{Strengths and Limitations}

A strong Report Card Advisory Committee with representation from across the country's academic institutions, as well as governmental sectors, participated in this study. A major limitation of the 2016 Portuguese Report Card was the lack of information in some of the assessed domains to provide objective information about the grades for each indicator. In the absence of robust data on some parameters, grades were assigned based on local studies and expert consensus. Most of the objective data came from scientific papers designed to study relationships and not to assess the monitoring of the Portuguese population as a whole. Thus, efforts should be made to seek information that might provide an understanding of PA of children and adolescents in Portugal.

\section{Conclusion}

Portuguese children and adolescents seemed to attain a low level of PA intensities, in addition to larger amounts of time spent in sedentary behaviors, compared with recommendations. Effective policies of PA promotion and implementation are needed in different domains of youngster's daily life.

\section{Acknowledgments}

The authors which to thanks Dr Paulo Rocha from IPDJ for making available a set of information that was need to complete this report. Mota was supported by grants FCT/ UID/DTP/00617/2013 and IPDJ-PNDpT 2016

\section{References}

1. Matos MG, Simões C, Camacho I, Reis M. Relatório do Estudo HBSC 2014-A Saúde dos Adolescentes Portugueses em Tempos de Recessão-Dados Nacionais do Estudo HBSC de 2014. Lisboa: Centro de Malária e Outras Doenças Tropicais/IHMT/UML and Faculdade de Motricidade Humana; 2015.

2. Baptista F, Santos DA, Silva AM, et al. Prevalence of the Portuguese population attaining sufficient physical activity. Med Sci Sports Exerc. 2012;44(3):466-473. PubMed doi:10.1249/MSS.0b013e318230e441

3. Directorate-General for Education and Culture. Special Eurobarometer 412-Sport and Physical Activity. Brussels: European Commission; 2014.

4. Rezende LF, Sá TH, Mielke GI, Viscondi JY, Rey-López JP, Garcia LM. All-cause mortality attributable to sitting time: analysis of 54 countries worldwide. Am J Prev Med. 2016;51(2):253-63. PubMed doi:10.1016/j.amepre.2016.01.022

5. Tremblay MS, Gray CE, Akinroye K, et al. Physical activity of children: a global matrix of grades comparing 15 countries. J Phys Act Health. 2014;(Suppl 1):S113-S125. PubMed doi:10.1123/jpah.11. s1.s113

6. Matos MG, Simões C, Tomé G, et al. Equipa Aventura Social. Aventura Social \& Saúde-A Saúde dos Adolescentes Portugueses-Relatório do Estudo HBSC 2010. Lisboa: Centro de Malária e Outras Doenças Tropicais/IHMT/UML and Faculdade de Motricidade Humana; 2012.

7. Baptista F, Silva AM, Santos DA, et al. Observatório Nacional da Atividade Física-Livro Verde da Atividade Física. Lisboa: Edições Instituto Desporto de Portugal; 2011.
8. World Health Organization (WHO). Global Recommendations on Physical Activity for Health. Geneva, Switzerland: World Health Organization; 2010.

9. Direção Geral de Educação (DGE). Relatório do Programa do Desporto Escolar 2009-2012. Lisboa: Divisão do Desporto Escolar da Direção Geral da Educação; 2012.

10. Instituto Português do Desporto e Juventude. Estatísticas do Desporto. Instituto Português do Desporto e Juventude. http://www.idesporto. pt/conteudo.aspx?id=103. Accessed April 2016.

11. Pizarro A, Santos MP, Ribeiro JC, Mota J. Physical activity and active transport are predicted by adolescents' different built environment perceptions. J Public Health. 2012;20:5-10. doi:10.1007/s10389011-0432-4

12. Pizarro A, Ribeiro JC, Marques EA, Mota J, Santos MP. Is walking to school associated with improved metabolic health?: a cross sectional study in 10 to 12 year old Portuguese children. Int J Behav Nutr Phys Act. 2013;10(1):12. PubMed doi:10.1186/1479-5868-10-12

13. Administração Regional de Saúde do Alentejo (ARSA). Estudo de Saúde da População Infantil da Região Alentejo - Relatório. Évora: Núcleo Regional do Alentejo da Plataforma contra a Obesidade da ARSA; 2013.

14. Pizarro A, Schipperijn J, Andersen HB, Ribeiro JC, Mota J, Santos MP. Active commuting to school in Portuguese adolescents: using PALMs to detect trips. J Transp Health. 2016. doi:10.1016/j.jth.2016.02.004

15. Cordovil R, Lopes F, Neto C. Children's (in)dependent mobility in Portugal. J Sci Med Sport. 2015;18(3):299-303. PubMed doi:10.1016/j. jsams.2014.04.013

16. Maitland C, Stratton G, Foster S, Braham R, Rosenberg M. The dynamic family home: a qualitative exploration of physical environmental influences on children's sedentary behaviour and physical activity within the home space. Int J Behav Nutr Phys Act. 2014;11:157-169. PubMed doi:10.1186/s12966-014-0157-1

17. Maia J, Gomes TN, Trégouet D-A, Katzmarzyk PT. Familial resemblance of physical activity levels in the Portuguese population. J Sci Med Sport. 2014;17:381-386. PubMed doi:10.1016/j. jsams.2013.09.004

18. Gomes TN, Dos Santos FK, Garganta RM, Kenny DA, Katzmarzyk PT, Maia JA. Multi-level modelling of physical activity in nuclear families. Ann Hum Biol. 2014;41(2):138-144. PubMed doi:10.3109 /03014460.2013.836243

19. Santos MP, Pizarro A, Mota J, Marques E. Parental physical activity, safety perceptions and children's independent mobility. BMC Public Health. 2013;13(1):584-590. PubMed doi:10.1186/1471-2458-13-584

20. Vale S, Ricardo N, Soares-Miranda L, Santos R, Moreira C, Mota J. Parental education and physical activity in pre-school children. Child Care Health Dev. 2014;40(3):446-452. PubMed doi:10.1111/ cch.12041

21. Silva P, Sousa M, Sá C, Ribeiro J, Mota J. Physical activity in high school during the "free-time" periods. Eur Phys Educ Rev. 2015;21:135-148. doi:10.1177/1356336X14555295

22. Loureiro N, Matos MG, Santos MM, Mota J, Diniz JA. Neighborhood and physical activities of Portuguese adolescents. Int J Behav Nutr Phys Act. 2010;7:33. PubMed doi:10.1186/1479-5868-7-33

23. Vitor Fontes Rodrigues. Ciclovias de Portugal. Ciclovia. http://www. ciclovia.pt/. Accessed April 2016.

24. IPDJ. Programa Nacional de Desporto para Todos [National Sports for All Programme]. Lisbon: Portuguese Institute of Youth and Sports (IPDJ); 2015.

25. Directorate-General of Health. National Health Plan 2004-2010. In: Directorate-General of Health, ed. Health Mo. Lisbon: Government of Portugal; 2004:101-112. 


\section{Erratum: Mota et al (2016)}

The second author's name was incorrectly listed as Manuel Joâo Coelho e Silva. The correct spelling is Manuel João Coelho-e-Silva. The online version has been corrected. 